\title{
Fricção Científica: \\ será necessária uma alternativa à revisão inter-pares em avaliações científicas?
}

\author{
MIGUEL A.R.B. C A S T A N H *
}

O trabalho científico, desde a ideia original até à publicação do trabalho final e impacto subsequente, é frequentemente caricaturado pela seguinte sucessão de etapas:
1) Entusiasmo
2) Desilusão
3) Pânico
4) Procura do culpado
5) Castigo dos inocentes
6) Honrà e glória para os não participantes

Para que a caricatura não se torne retrato e se atinja um nível pleno de verdade, honestidade e satisfação na actividade científica, há que manter o ponto 1) e abolir os restantes (excepto o $4^{\circ}$ ), se for caso disso). Impetir que a eventual honra e glória (que na quase totalidade das vezes é desprovida de qualquer reconhecimento público ou fausto material) vá parar a mãos e cabeças não participantes, tem solução relativamente fácil: abolir a co-autoria honorária de artigos científicos. As relações hierárquicas são distintas das colaborações científicas. Só deve constar como autor quem participou de facto no desenvolvimento do trabalho que se publica. É este o único critério que se pode seguir com honestidade. O uso de co-autoria como compensação de outras actividades, para contentar superiores hierárquicos ou em função da pressão destes, é condenável [1]. Outros problemas não são de solução tão simples: Como impedir que a mediocridade se generalize nas publicações científicas sem travar a inovação? O actual mecanismo de triagem existente nas revistas científicas de reconhecido valor, baseia-se na avaliação por peritos que emitem pareceres sobre determinado trabalho. Desse parecer pode resultar, ou não, a publicação, segundo decisão do Corpo Editorial da revista. Os peritos escolhidos como revisores permanecem anónimos para os autores ${ }^{1}$. O sistema tem a designação habitual de Peer-review (revisão inter-pares) e não é perfeito. As lacunas são facilmente apontadas:
1) Atrasa a publicação dos trabalhos, tornando o processo antecedente à publicação, por vezes, bastante fastidioso. O problema de atraso de publicação exerce tal pressão sobre os corpos editoriais das revistas, que a Sociedade Americana de Química decidiu colocar em edição electrónica todas as suas 26 publicações, imediatamente após o processo de revisão inter-pares, desde o último mês de Setembro [2]. Alguns defendem mesmo que os manuscritos sejam submetidos a diferentes revistas simultaneamente [3], mas tais medidas acarretam ris$\cos$ (nomeadamente, a multiplica- ção de versões do mesmo trabalho) e existem outros mecanismos, mais rápidos, de apresentação de resultados e conclusões (congressos, divulgação electrónica de resumos, etc.) que tornam as submissões simultâneas desnecessárias [4].

2) Aumenta os custos associados à publicação ${ }^{2}$,

3) É vulnerável a condutas contrárias à ética, como plágio, quebra de confidencialidade e boicotes por parte de grupos/investigadores concorrentes. O sistema de avaliação dos candidatos a bolsas de estudo ou de projectos científicos candidatos a financiamento, consiste também

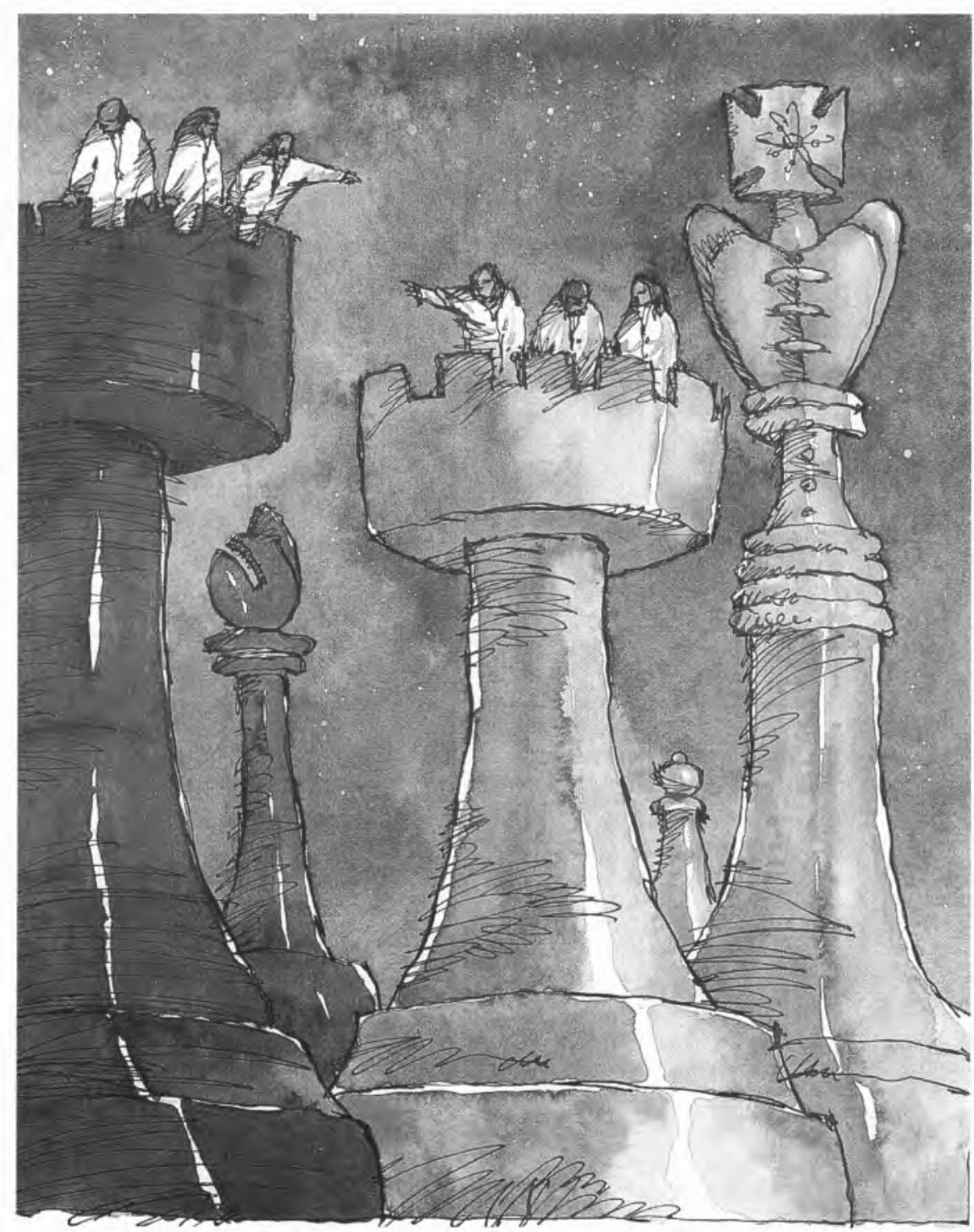


numa revisão inter-pares, tal como se usa para julgar o mérito de um trabalho científico submetido a uma revista de prestígio para publicação. Duas investigadoras suecas realizaram um estudo sobre a atribuição de fundos para bolsas durante o ano de 1995 no seu país e concluíram que os candidatos beneficiam das ligações aos revisores [5]. No entanto, em países de maior dimensão este efeito de "interacção com o vizinho mais próximo" pode não ser tão forte $[6,7]$.

4) O cepticismo e/ou ignorância dos revisores pode impedir a publicação de trabalhos que contenham visões mais inovadoras. Pelo menos oito artigos capazes de merecer o prémio Nobel para os seus autores foram rejeitados por revisores [8].

Mas é um facto que este sistema tem impedido uma avalanche de mediocridade grosseira na literatura científica. É fácil de imaginar o que aconteceria se não existisse qualquer tipo de crivo sobre a qualidade dos trabalhos a publicar: curas milagrosas, materiais fantásticos, extraterrestres, monstros em lagos ou fora deles, e tudo o mais em que se queira crer. A falta de credibilidade generalizar-se-ia. Passaríamos a ter Ficção Científica a disputar espaço à Ciência. Mais vale a actual fricção científica que a alternativa ficção científica. A questão é i) como melhorar o actual sistema? ou ii) qual o sistema que pode substituir o actual nas funções de crivo da qualidade dos trabalhos enviados às revistas para publicação? Este tema tem suscitado a atenção crescente da comunidade científica, incluindo a ligada à Química. Nos últimos três anos, vários artigos de opinião têm sido publicados. O assunto tem vindo a tomar uma actualidade tal que ultrapassou as barreiras herméticas do seio da comunidade científica; algo de muito raro para um assunto que não tem implicações directas com o quotidiano do cidadão comum. A revista Ambassador, no seu número de Setembro de 1997 dedicou um artigo a este tema [9]. A National Science Foundation (EUA), pressionada por críticas constantes, nomeou uma comissão para estudar a simplificação do processo de avaliação de projectos candidatos a financiamento, sucedendo ao processo de revisāo inter-pares. O anterior sistema foi substituído a 1 de Outubro deste ano, mas as alterações resumem-se à inclusão de parâmetros de relevância económica e social [10].

\section{ALTERNATIVAS PROPOSTAS PARA SUCEDER AO SISTEMA DE REVISÃO INTER-PARES}

Rustum Roy, um guru dos críticos ao sistema de revisão interpares e investigador na Pennsylvania State University, deu nas vistas por anunciar em conferência de imprensa, em 1992, os resultados da sua investigação sobre síntese de diamantes, antes destes serem aceites para publicação numa revista científica. Desprezou a "certificação" que os seus pares lhe poderiam dar [11]. Em 1995 criou uma revista para publicar rapidamente resultados novos, inesperados e inovadores, sem o sistema de revisão interpares. A revista, intitulada Innovations in Materials Research, examina os curricula dos autores, nomeadamente as suas publicações passadas [12]. Qualquer investigador com pelo menos 50 publicações ou patentes. pode publicar livremente nesta revista. Se os autores ainda não tiverem 50 artigos ou patentes, podem ser patrocinados por colegas nestas condições. Se isso não for possível, os autores têm que deixar a sua lista de publicações passadas ser avaliada. Ver neste sistema uma alternativa ao da revisão inter-pares é claramente um equívoco. Para possuir um curriculum com publicações é obrigatório ter passado inicialmente por uma fase de revisão inter-pares. Corolário: o sistema de revisão inter-pares tem que existir. Não há alternativa. Se não se chegar a 50 publicações, é necessário um "pa- trocínio". se o sistema de revisões inter-pares é vulnerável ao compadrio, o de "patrocínio", vive dele. Em último recurso, a lista de publicações dos autores é avaliada pelo corpo editorial. Isto é, a revista tem o seu próprio sistema de revisão inter-pares, só que recaindo sobre uma matéria diferente. Mas talvez o mais assustador seja pensar que quem atingir 50 publicações (muito fácil no sistema proposto, com uma "mãozinha" de um colega que já as tenha...) pode publicar indiscriminadamente, sem controlo ${ }^{3}$. Apesar de todas estas críticas, em que não estou só [13], Rustum Roy tem defensores [14, 15].

Mais consistente é a proposta de Juan M. Campanario [8]. No sistema de publicação actual, os autores competem para obterem "espaço de publicação" em revistas prestigiadas, enquanto outras revistas, de menor prestígio, têm dificuldades em obter manuscritos e podem ter que aceitar trabalhos de menor qualidade para se manterem activas. Segundo aquele investigador da Universidade de Alcalá (Madrid), a solução seria a criação de uma "montra virtual", onde os autores colocariam os seus manuscritos. Os corpos editoriais das revistas poderiam então consultar periodicamente esta "montra" para localizar manuscritos potencialmente inovadores. Se mais que uma oferta for feita aos autores, estes escolherão em que revista publicar. As revistas recrutariam especialistas em cada campo com a função de "caça-talentos" (neste caso, não pessoas mas manuscritos). A ideia é que com vários "caça-talentos" à procura de trabalhos meritórios, a probabilidade de um manuscrito com ideias inovadoras não ortodoxas ser atrasado por um único revisor preconceituoso, seria quase nula. Em alguns casos, os autores prefeririam uma publicação mais rápida, em revistas menos prestigiadas. Este sistema, contudo, não contorna a revisão inter-pares clássica, apenas reverte os papéis da oferta $e$ procura de manuscritos. Não se trata de uma alteração de princípio, 
tão só de uma mudança na praxis da implementação do sistema de revisão inter-pares, com vantagens quanto ao tempo de publicação e sensibilidade a preconceitos individuais dos revisores. Na realidade, uma meditação mais atenta quanto a esta proposta, revela que ela não é diferente da submissão simultânea de um manuscrito a várias revistas, a que já aludi antes.

\section{NECESSITAMOS DE MUDAR?}

Que o sistema actual pode ser permeável ao preconceito, ignorância e conservadorismo, ninguém duvida. No entanto, é inegável que a grande oferta de revistas onde publicar permite contornar estas desvantagens. Além disso, entre o fogo cerrado e as críticas, poucos apontam as virtudes do sistema. É experiência comum a muitos que bastantes revisores apresentam críticas construtivas e oferecem sugestões úteis que podem elevar a qualidade do manuscrito, revertendo o benefício em favor dos autores e leitores. Para isso, há que escolher bons revisores. Esta é uma missão dos editores das revistas, que deveriam assumir um papel de grande relevo e total responsabilidade. A questão que se coloca, no entanto, é: como garantir que a ignorância (muitas vezes disfarçada de arrogância e má-educação traduzidas em comentários insultuosos na apreciação dos manuscritos [16]) e má fé estejam ausentes? Obviamente, grande parte do problema seria resolvido pela auto-exclusão de cada um, se não se sente com competência para avaliar um determinado trabalho. Mas convém haver um mecanismo institucional; algo que não recaia tanto sobre a sempre falível natureza humana. Estamos condenados a encarar a submissão simultânea de manuscritos a várias revistas como única forma de minorar os defeitos do sistema de revisão inter-pares? Creio que não. Existe uma alternativa mais simples e menos arriscada.

\section{UM MAL DESNECESSÁRIO: A REVISÃO ANÓNIMA DE CONTRIBUIÇÕES CIENTÍFICAS}

Em 4 de Julho de 1994, o Prof, Gregorio Weber ${ }^{4}$, enviou uma carta ao editor da revista Nature (que provavelmente nunca chegou a ser publicada), intitulada $A n$ unnecessary evil: the anonymous refereeing of scientific contributions. Nessa carta, é dito que "I have seen example after example in which the report of the referee, if such is the appropriate designation, consists of a long diatribe with many adjectives and few nouns and verbs. The object of the exercise appears to be to make clear, without benefit or further proof, the superiority of the referee or the dismal inferiority of the author. Habit has apparently immunized editorial officers against the sight and sound of such horrors as indicated by the accompanying little note that, " ... in view of the unfavorable report of the referee we have regrettably to deny publication." I feel few doubts that if the same referee would have known that his statements were to became public record he would think twice before substituting real substantive argumentation by value judgments and pejorative adjectives. In my scientific life I have refereed hundreds of papers, and no doubt I made some erroneous statements, but I always took special care to limit my reports to what I considered scientific arguments. I have kept copies of most of those reports, and in no case I would have in the least objected that my name be communicated to the author of the criticized paper." Aqui está uma boa solução: abolir o anonimato do sistema de revisão inter-pares. Quais são os argumentos que podem justificar o anonimato se a aceitação ou rejeição de artigos (ou concessão ou não de bolsas e financiamentos) só pode basear-se em critérios de natureza científica? Da transparência do processo só podem resultar benefícios, vindos da responsabilização dos revisores. Linguagem ofensiva, má-fé e falta de objectividade e conteúdo científico seriam claramente diminuídos em textos não anónimos. Igualmente, um revisor sob protecção do anonimato é menos sensível a combater o seu próprio desleixo e pode, consequentemente, demorar um tempo injustificadamente longo para efectuar a sua tarefa. Não necessitamos rejeitar o sistema de revisão inter-pares, basta melhorá-lo removendo o secretismo. A esta conclusão chegaram também Wenneras e Wold em resultado dos estudos atrás citados. A ideia que seria mais difícil recrutar revisores caso o anonimato fosse banido, é desmentida pela prática (ainda que verdadeira, poderíamos argumentar que restariam menos mas melhores revisores). Na Suécia, uma decisão de um tribunal obrigou a tornar públicos os resultados das avaliações dos revisores, desde 1995 , mas não houve debandadas nem deserções em larga escala das comissões de avaliação. Esta questão deveria ser ponderada por todos os responsáveis pela avaliação do desempenho na actividade científica, quer se trate da publicação de manuscritos, atribuição de bolsas ou financiamento de projectos. Tal como escreveu ainda o Prof. G. Weber na carta há pouco referida, "Secrecy and anonymity have been consistently driven off from many aspects of public life where they were once enshrined. Their disappearance has not been followed by any bad effects but, on the contrary, by renewed confidence in the institutions that have banished them. Science in general, and scientific publication in particular, have much to gain by abandoning the old "star chamber" methods in favor of the public transparency which is in the very nature of science" Eu concordo! E o leitor?

\footnotetext{
* Centro de Química Física Molecular (Instituto Superior Técnico, Lisboa) e Departamento de Quimica e Bioquímica (Faculdade de Ciências da UL, Lisboa)
}

\section{NOTAS}

1 Em alguns paises e em determinadas áreas (como, por exemplo a de Direito, nos EUAI, existe um anonimato reciproco: os revisores permanecem anónimos para os autores e vice-versa. 


\begin{abstract}
2 Estes custos não estão relacionados com o pagamento aos revisores, uma vez que o trabalho de avaliação nāo é pago. Os custos implicados incluem portes de correio, apoio de secretariado e material gasto na reformulação de manuscritos, execução de experiências adicionais, etc.
\end{abstract}

${ }^{3}$ Faz-me lembrar uma análise descrita por I. Ziman em Reliable Knowledge (Cambridge UP, 1978): " A very typical case is the professional scientist of some stan. ding who goes gently around the curve and begins to expound some incoherent irrational theory. (...) It is very difficult to prove that he is insane. When a bank manager goes dotty, and hands out five pound notes in the street, claiming to be the reincarnation of the late Mr. Paul Getty, there is not much doubt that he is unfit for his job; but a famous professor respected for his brilliant insights, long experienced in the byeways of his subject, can conjure up a fascinating scaffolding of special pleading and plausible conjecture to supporte his obsession. The only professional colleagues competent to diagnose his condition can be accused or prejudice or jealousy., Este investigador publicaria indefinidamente na revista criada por Rustum Roy.

${ }^{4}$ Nota biográfica em Química - Boletim Soc. Port. Quimica, 66, (1997).

\section{REFERÊNCIAS BIBLIOGRÁFICAS}

1. Santos, M. N. B. (1988) A desonestidade em Ciência, Boletim da Soc. Portuguesa de Química, 31 (Série II) Março, 9

2. Schulz, W. (1997) ACS moves to full on-line publishing, Chem. \& Eng. News, 18 Agosto, 9.

3. Berezin, A. (1997) Journal submission, Chem. \& Eng. News, 24 Março, 6.

4. Bauer, S. H. (1997) Establishing priority, Chem. \& Eng. News, 4 Agosto, 6

5. Wenneras, C. e Wold, A. (1997) Nepotism and sexism in peer-review, Nature, 387, 341 .

6. Roubi, M. (1997) Study suggests Swedish peer review biased, Chem. \& Eng. News, Maio, 10.

7. Lepkowski, W. (1997) No gender bias in its grant awards, says NSF, Chem. \& Eng. News., 8 Setembro, 10.

8. Campanario, I. M. (1997) The "journal scout", The Scientist, 11(10), 9.
9. Daviss, B. (1997) The referees of R\&D, Ambassador. Setembro, 32-36

10. Lepkowski, W. (1997) Peer review adds social relevancy, Chem. \& Eng. News, 7 Abril, 9.

11. Borman, S. (1992) Solid-state process produces synthetic diamond, Chem. \& Eng. News, 26 Outubro, 5 .

12. Dagani, R. (1995) New journal forgoes traditional peer review, Chem, \& Eng. News, 22 Maio, 26-27

13. e. g. Robinson, C. W. (1995) Scrapping peer review, Chem. \& Eng. News, 17 Julho, 4.

14. Erhan, S. (1995) Scrapping peer review, Chem. \& Eng. News, 17 Julho, 4

15. Evans, M. (1996) Peer review, Chem. \& Eng. News, 29 laneiro, 4

16. Davenport, D.A. (1996) Opprobrium: Occurrence, Preparation, Properties, and Uses, The Chemical Intelligencer, Abril, 45-50.

\section{MOBILIÁRIO ESPECÍFICO PARA LABORATÓRIOS}

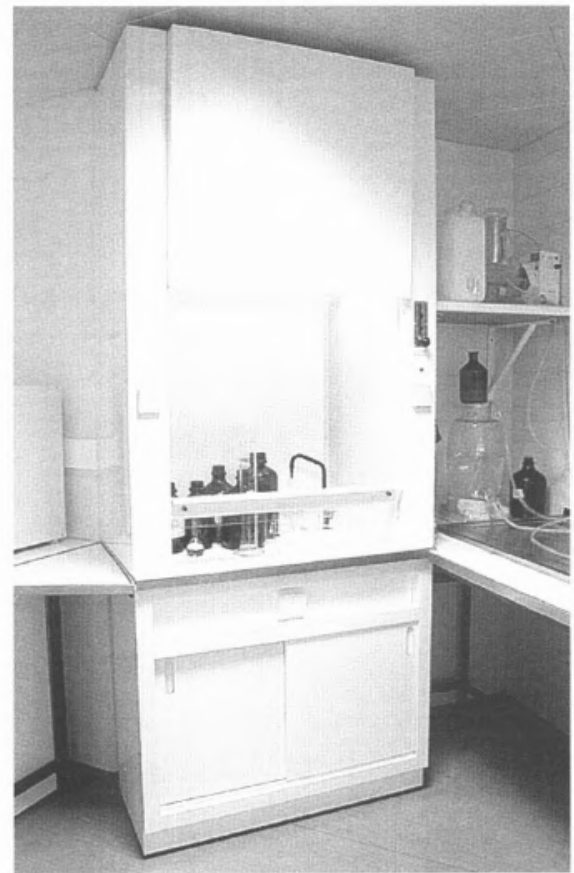

Bancadas Murais

Bancadas Centrais

Bancadas de Lavagem

Hottes

Mesas para Balanças

Carros para Transporte

Armários de Parede

Armários Ventilados para Reagentes

\section{Sociedade de Representações e Química, Lda.}

Rua Coronel Santos Pedroso, 15 • 1500 LISBOA • Tel.: 7165160 • Fax: 7165169 Sede Social: Av. da Liberdade, 220-2 $2^{\circ} 129$ LISBOA CODEX Rua 5 de Outubro, 269 • 4100 PORTO • Tels.: 6093069 • Fax: 6000834 SOQUÍMICA Email: soquimica@mail.telepac.pt; Internet: www.soquimica.pt 